Acta Crystallographica Section E

Structure Reports

Online

ISSN 1600-5368

\section{Hazel S. Wilkinson and William T. A. Harrison*}

Department of Chemistry, University of Aberdeen, Meston Walk, Aberdeen AB24 3UE, Scotland

Correspondence e-mail:

w.harrison@abdn.ac.uk

\section{Key indicators}

Single-crystal X-ray study

$T=293 \mathrm{~K}$

Mean $\sigma(\mathrm{C}-\mathrm{C})=0.003 \AA$

$R$ factor $=0.024$

$w R$ factor $=0.064$

Data-to-parameter ratio $=33.3$

For details of how these key indicators were automatically derived from the article, see http://journals.iucr.org/e.

\title{
tert-Butylammonium dihydrogenarsenate
}

The title compound, $\left(\mathrm{C}_{4} \mathrm{H}_{12} \mathrm{~N}\right)\left[\mathrm{H}_{2} \mathrm{AsO}_{4}\right]$, contains a network of tert-butylammonium cations and dihydrogenarsenate anions $\left[d_{\mathrm{av}}(\mathrm{As}-\mathrm{O})=1.682(2) \AA\right]$. The crystal packing involves $\mathrm{N}-\mathrm{H} \cdots \mathrm{O}\left[d_{\mathrm{av}}(\mathrm{H} \cdots \mathrm{O})=1.96 \AA, \theta_{\mathrm{av}}(\mathrm{N}-\mathrm{H} \cdots \mathrm{O})=\right.$ $169^{\circ}$ and $\left.d_{\text {av }}(\mathrm{N} \cdots \mathrm{O})=2.837(3) \AA\right]$ and $\mathrm{O}-\mathrm{H} \cdots \mathrm{O}$ $\left[d_{\mathrm{av}}(\mathrm{H} \cdots \mathrm{O})=1.68 \AA, \theta_{\mathrm{av}}(\mathrm{O}-\mathrm{H} \cdots \mathrm{O})=169^{\circ}\right.$ and $d_{\mathrm{av}}(\mathrm{O} \cdots \mathrm{O})$ $\AA]$ hydrogen bonds, resulting in a layered structure.

\section{Comment}

The title compound, (I) (Fig. 1), was prepared as part of our ongoing structural studies of hydrogen-bonding interactions in protonated amine (di)hydrogen arsenates (Lee \& Harrison, $2003 a, b, c)$.

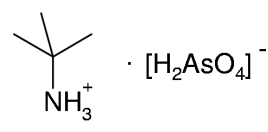

(I)

The $\left[\mathrm{H}_{2} \mathrm{AsO}_{4}\right]^{-}$dihydrogenarsenate group in (I) shows its normal tetrahedral geometry $\left[d_{\text {av }}(\mathrm{As}-\mathrm{O})=1.682(2) \AA\right]$, with the protonated $\mathrm{As} 1-\mathrm{O} 3$ and $\mathrm{As} 1-\mathrm{O} 4$ vertices showing their expected lengthening relative to the unprotonated $\mathrm{As}-\mathrm{O}$ bonds, which have formal partial double-bond character (Table 1). The tert-butylammonium cation shows no unusual geometrical features.

As well as electrostatic attractions, the component species in (I) interact by means of a network of cation-to-anion $\mathrm{N}-\mathrm{H} \cdots \mathrm{O}$ and anion-to-anion $\mathrm{O}-\mathrm{H} \cdots \mathrm{O}$ hydrogen bonds (Table 2). The $\left[\mathrm{H}_{2} \mathrm{AsO}_{4}\right]^{-}$units are linked into polymeric chains propagating along [010] by way of inversion-generated pairs of $\mathrm{O}-\mathrm{H} \cdots \mathrm{O}$ bonds, alternately involving $\mathrm{O} 3-\mathrm{H} 1 \cdots \mathrm{O} 1$

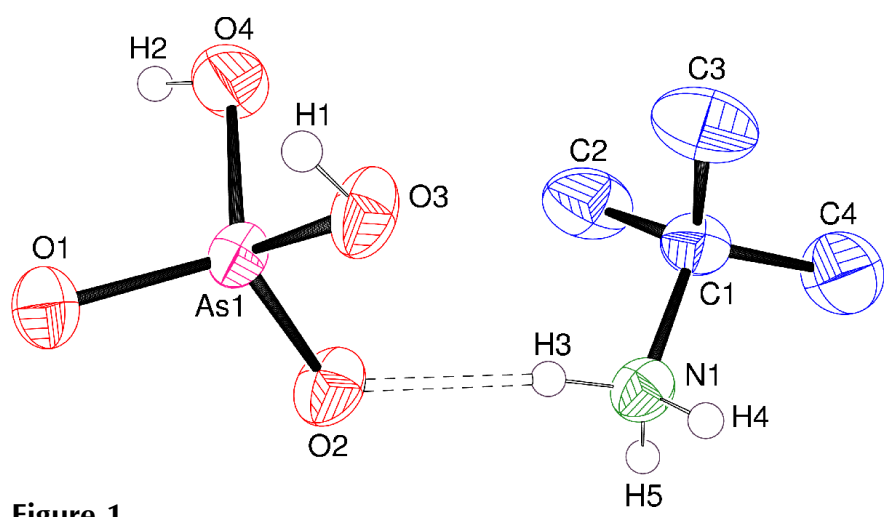

Figure 1

The asymmetric unit of (I) (50\% displacement ellipsoids). $\mathrm{H}$ atoms are drawn as small spheres of arbitrary radius and the hydrogen bond is indicated by a dashed line. $\mathrm{C}-\mathrm{H} \mathrm{H}$ atoms have been omitted for clarity.
Received 28 July 2004

Accepted 25 August 2004

Online 4 September 2004
(C) 2004 International Union of Crystallography Printed in Great Britain - all rights reserved 


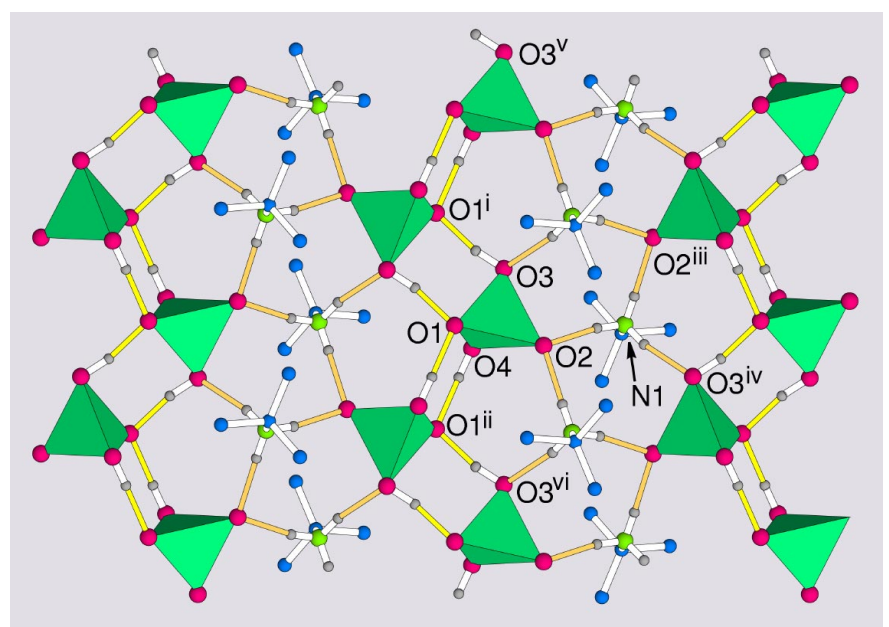

Figure 2

Detail of a hydrogen-bonded dihydrogenarsenate/tert-butylammonium sheet in (I). Colour key: $\left[\mathrm{H}_{2} \mathrm{AsO}_{4}\right]^{-}$tetrahedra green, $\mathrm{O}$ atoms red, $\mathrm{H}$ atoms grey, $\mathrm{C}$ atoms blue, $\mathrm{N}$ atoms green (all radii arbitrary). The $\mathrm{H} \cdots \mathrm{O}$ portions of the $\mathrm{O}-\mathrm{H} \cdots \mathrm{O}$ and $\mathrm{N}-\mathrm{H} \cdots \mathrm{O}$ hydrogen bonds are highlighted in yellow and orange, respectively. Symmetry codes as in Table 2; additionally, (v) $\mathrm{x}, y-1, z ;$ (vi) $x, 1+y, z$.

and $\mathrm{O} 4-\mathrm{H} 2 \cdots \mathrm{O} 1$ links (Fig. 2). This results in every $\left[\mathrm{H}_{2} \mathrm{AsO}_{4}\right]^{-}$tetrahedron in the chain making one hydrogen bond to each of its neighbours and accepting one hydrogen bond from each neighbour. The As...Ass $\left(v i a \mathrm{O} 3-\mathrm{H} 1 \cdots \mathrm{O} 1^{\mathrm{i}}\right)$ and $\mathrm{As} \cdots \mathrm{As}^{\mathrm{ii}}\left(\right.$ via $\mathrm{O} 4-\mathrm{H} 2 \cdots \mathrm{O} 1^{\mathrm{ii}}$ ) separations are 4.3002 (4) and 4.2662 (3) $\AA$, respectively (see Table 2 for symmetry codes). Similar hydrogen-bonded chains of $\left[\mathrm{H}_{2} \mathrm{AsO}_{4}\right]^{-}$anions have been seen in piperidinum dihydrogenarsenate, $\left(\mathrm{C}_{5} \mathrm{H}_{12} \mathrm{~N}\right)\left[\mathrm{H}_{2} \mathrm{AsO}_{4}\right]$ (Lee \& Harrison, 2003b), although in this case they are generated by a $2_{1}$ screw axis.

As shown in Table 2, the organic species interacts with the dihydrogenarsenate chains by way of three $\mathrm{N}-\mathrm{H} \cdots \mathrm{O}$ hydrogen bonds $\left[d_{\mathrm{av}}(\mathrm{H} \cdots \mathrm{O})=1.96 \AA \theta_{\mathrm{av}}(\mathrm{N}-\mathrm{H} \cdots \mathrm{O})=169^{\circ}\right.$ and $\left.d_{\mathrm{av}}(\mathrm{N} \cdots \mathrm{O})=2.837(3) \AA\right]$, such that each tert-butylammonium cation cross-links a dihydrogenarsenate chain to its neighbour by forming two hydrogen bonds to one chain, and one to the other. This results in neutral (101) layers (Fig. 3) of stoichiometry $\left(\mathrm{C}_{4} \mathrm{H}_{12} \mathrm{~N}\right)\left[\mathrm{H}_{2} \mathrm{AsO}_{4}\right]$, which interact with each other by van der Waals forces.

\section{Experimental}

An aqueous tert-butylamine solution $(10 \mathrm{ml}$ of $0.5 \mathrm{M})$ was added to a $\mathrm{H}_{3} \mathrm{AsO}_{4}$ solution $(10 \mathrm{ml}$ of $0.5 \mathrm{M})$, resulting in a clear solution. A mass of plate-shaped and rod-like crystals of (I) grew as the water evaporated over the course of a few days.

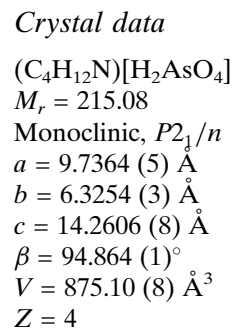

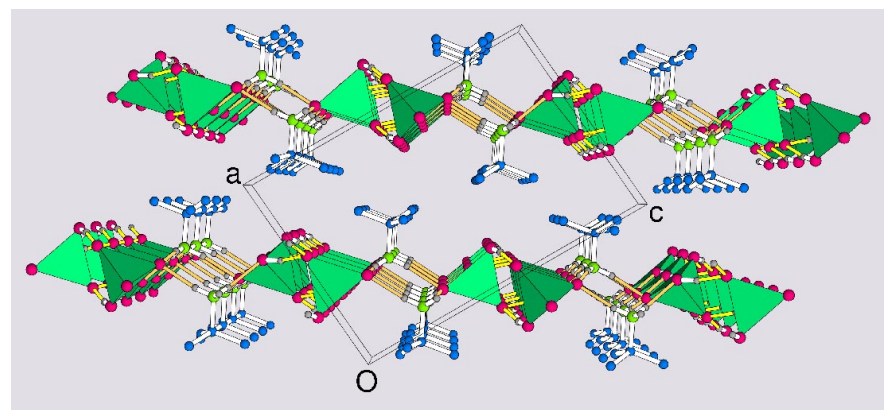

Figure 3

Projection of (I) onto (010). The colour key is as in Fig. 2.

\section{Data collection}

Bruker SMART1000 CCD diffractometer

$\omega$ scans

Absorption correction: multi-scan (SADABS; Bruker, 1999)

$T_{\min }=0.226, T_{\max }=0.831$

8742 measured reflections

\section{Refinement}

Refinement on $F^{2}$

$R\left[F^{2}>2 \sigma\left(F^{2}\right)\right]=0.024$

$w R\left(F^{2}\right)=0.064$

$S=0.88$

3165 reflections

95 parameters

Table 1

Selected bond distances $(\AA)$.

\begin{tabular}{llll}
\hline As1-O2 & $1.6412(13)$ & As1-O3 & $1.7061(13)$ \\
As1-O1 & $1.6687(13)$ & As1-O4 & $1.7101(14)$ \\
\hline
\end{tabular}

Table 2

Hydrogen-bonding geometry $\left(\AA{ }^{\circ}\right)$.

\begin{tabular}{lllll}
\hline$D-\mathrm{H} \cdots A$ & $D-\mathrm{H}$ & $\mathrm{H} \cdots A$ & $D \cdots A$ & $D-\mathrm{H} \cdots A$ \\
\hline $\mathrm{O} 3-\mathrm{H} 1 \cdots \mathrm{O} 1^{\mathrm{i}}$ & 0.97 & 1.62 & $2.5752(19)$ & 166 \\
$\mathrm{O} 4-\mathrm{H} 2 \cdots 1^{\text {ii }}$ & 0.94 & 1.74 & $2.6763(19)$ & 172 \\
$\mathrm{~N} 1-\mathrm{H} 3 \cdots \mathrm{O} 2$ & 0.89 & 1.92 & $2.801(2)$ & 168 \\
$\mathrm{~N} 1-\mathrm{H} 4 \cdots \mathrm{O} 2^{\text {iii }}$ & 0.89 & 1.88 & $2.765(2)$ & 173 \\
$\mathrm{~N} 1-\mathrm{H} 5 \cdots 3^{\text {iv }}$ & 0.89 & 2.07 & $2.944(2)$ & 166 \\
\hline
\end{tabular}

Symmetry codes: (i) $-x,-y, 1-z$; (ii) $-x, 1-y, 1-z$; (iii) $\frac{1}{2}-x, y-\frac{1}{2}, \frac{1}{2}-z$; (iv) $\frac{1}{2}-x, \frac{1}{2}+y, \frac{1}{2}-z$.

The $\mathrm{O}-\mathrm{H} \mathrm{H}$ atoms were found in difference maps and refined by riding on their carrier $\mathrm{O}$ atoms in their as-found relative positions. $\mathrm{H}$ atoms bonded to $\mathrm{C}$ and $\mathrm{N}$ atoms were placed in calculated positions $[\mathrm{d}(\mathrm{C}-\mathrm{H})=0.96 \AA$ and $\mathrm{d}(\mathrm{N}-\mathrm{H})=0.89 \AA]$ and refined as riding, with the rigid $\mathrm{NH}_{3}$ or $\mathrm{CH}_{3}$ groups allowed to freely rotate about the bond joining the atoms in question to atom $\mathrm{C} 1$. The constraint $U_{\text {iso }}(\mathrm{H})=$ $1.2 U_{\text {eq }}\left(\mathrm{O}\right.$ or $\mathrm{N}$ parent atom) or $1.5 U_{\text {eq }}$ (methyl $\mathrm{C}$ parent atom) was applied as appropriate.

Data collection: SMART (Bruker, 1999); cell refinement: SAINTPlus (Bruker, 1999); data reduction: SAINT-Plus; program(s) used to solve structure: SHELXS97 (Sheldrick, 1997); program(s) used to refine structure: SHELXL97; molecular graphics: ORTEP-3 (Farrugia, 1997) and ATOMS (Shape Software, 1999); software used to prepare material for publication: SHELXL97. 


\section{metal-organic papers}

HSW thanks the Carnegie Trust for the Universities of Scotland for an undergraduate vacation studentship.

\section{References}

Bruker (1999). SMART (Version 5.624), SAINT-Plus (Version 6.02A) and $S A D A B S$. Bruker AXS Inc., Madison, Wisconsin, USA.
Farrugia, L. J. (1997). J. Appl. Cryst. 30, 565.

Lee, C. \& Harrison, W. T. A. (2003a). Acta Cryst. E59, m739-m741.

Lee, C. \& Harrison, W. T. A. (2003b). Acta Cryst. E59, m959-m960.

Lee, C. \& Harrison, W. T. A. (2003c). Acta Cryst. E59, m1151-m1153.

Shape Software (1999). ATOMS. Shape Software, 525 Hidden Valley Road, Kingsport, Tennessee, USA.

Sheldrick, G. M. (1997). SHELXL97. University of Göttingen, Germany. 


\section{supporting information}

Acta Cryst. (2004). E60, m1359-m1361 [https://doi.org/10.1107/S1600536804020963]

\section{tert-Butylammonium dihydrogenarsenate}

\section{Hazel S. Wilkinson and William T. A. Harrison}

tert-Butylammonium dihydrogenarsenate

Crystal data

$\left(\mathrm{C}_{4} \mathrm{H}_{12} \mathrm{~N}\right)\left[\mathrm{H}_{2} \mathrm{AsO}_{4}\right]$

$M_{r}=215.08$

Monoclinic, $P 2_{1} / n$

Hall symbol: -P 2 yn

$a=9.7364(5) \AA$

$b=6.3254(3) \AA$

$c=14.2606(8) \AA$

$\beta=94.864(1)^{\circ}$

$V=875.10(8) \AA^{3}$

$Z=4$

\section{Data collection}

Bruker SMART1000 CCD diffractometer

Radiation source: fine-focus sealed tube Graphite monochromator $\omega$ scans

Absorption correction: multi-scan

(SADABS; Bruker, 1999)

$T_{\min }=0.226, T_{\max }=0.831$

Refinement

Refinement on $F^{2}$

Least-squares matrix: full

$R\left[F^{2}>2 \sigma\left(F^{2}\right)\right]=0.024$

$w R\left(F^{2}\right)=0.064$

$S=0.88$

3165 reflections

95 parameters

0 restraints

Primary atom site location: structure-invariant direct methods
$F(000)=440$

$D_{\mathrm{x}}=1.633 \mathrm{Mg} \mathrm{m}^{-3}$

Mo $K \alpha$ radiation, $\lambda=0.71073 \AA$

Cell parameters from 3025 reflections

$\theta=2.4-32.4^{\circ}$

$\mu=3.85 \mathrm{~mm}^{-1}$

$T=293 \mathrm{~K}$

Bar, colourless

$0.55 \times 0.15 \times 0.05 \mathrm{~mm}$

8742 measured reflections

3165 independent reflections

1807 reflections with $I>2 \sigma(I)$

$R_{\text {int }}=0.023$

$\theta_{\max }=32.5^{\circ}, \theta_{\min }=2.4^{\circ}$

$h=-10 \rightarrow 14$

$k=-7 \rightarrow 9$

$l=-21 \rightarrow 21$

Secondary atom site location: difference Fourier map

Hydrogen site location: difmap $(\mathrm{O}-\mathrm{H})$ and geom (C-H and $\mathrm{N}-\mathrm{H})$

$\mathrm{H}$-atom parameters constrained

$w=1 /\left[\sigma^{2}\left(F_{\mathrm{o}}^{2}\right)+(0.0332 P)^{2}\right]$

where $P=\left(F_{0}^{2}+2 F_{\mathrm{c}}^{2}\right) / 3$

$(\Delta / \sigma)_{\max }=0.001$

$\Delta \rho_{\max }=0.47 \mathrm{e} \AA^{-3}$

$\Delta \rho_{\min }=-0.51$ e $\AA^{-3}$

\section{Special details}

Geometry. All e.s.d.'s (except the e.s.d. in the dihedral angle between two 1.s. planes) are estimated using the full covariance matrix. The cell e.s.d.'s are taken into account individually in the estimation of e.s.d.'s in distances, angles and torsion angles; correlations between e.s.d.'s in cell parameters are only used when they are defined by crystal symmetry. An approximate (isotropic) treatment of cell e.s.d.'s is used for estimating e.s.d.'s involving 1.s. planes. 
Refinement. Refinement of $F^{2}$ against ALL reflections. The weighted $R$-factor $w R$ and goodness of fit $S$ are based on $F^{2}$, conventional $R$-factors $R$ are based on $F$, with $F$ set to zero for negative $F^{2}$. The threshold expression of $F^{2}>\sigma\left(F^{2}\right)$ is used only for calculating $R$-factors(gt) etc. and is not relevant to the choice of reflections for refinement. $R$-factors based on $F^{2}$ are statistically about twice as large as those based on $F$, and $R$ - factors based on ALL data will be even larger.

Fractional atomic coordinates and isotropic or equivalent isotropic displacement parameters $\left(\AA^{2}\right)$

\begin{tabular}{lllll}
\hline & $x$ & $y$ & $z$ & $U_{\text {iso }}^{*} / U_{\text {eq }}$ \\
\hline As1 & $0.096480(18)$ & $0.25182(3)$ & $0.428460(11)$ & $0.02877(6)$ \\
O1 & $-0.07070(14)$ & $0.2606(2)$ & $0.44605(10)$ & $0.0360(3)$ \\
O2 & $0.13620(15)$ & $0.3528(2)$ & $0.32825(9)$ & $0.0424(4)$ \\
O3 & $0.15368(15)$ & $-0.0032(2)$ & $0.43072(9)$ & $0.0402(3)$ \\
H1 & 0.1102 & -0.0863 & 0.4773 & $0.048^{*}$ \\
O4 & $0.18923(15)$ & $0.3676(3)$ & $0.52194(10)$ & $0.0482(4)$ \\
H2 & 0.1545 & 0.5006 & 0.5378 & $0.058^{*}$ \\
N1 & $0.38027(16)$ & $0.2563(2)$ & $0.24651(10)$ & $0.0334(3)$ \\
H3 & 0.3062 & 0.2725 & 0.2783 & $0.040^{*}$ \\
H4 & 0.3824 & 0.1249 & 0.2243 & $0.040^{*}$ \\
H5 & 0.3766 & 0.3474 & 0.1988 & $0.040^{*}$ \\
C1 & $0.5091(2)$ & $0.2971(3)$ & $0.31140(15)$ & $0.0380(5)$ \\
C2 & $0.4956(3)$ & $0.5164(4)$ & $0.35241(18)$ & $0.0608(7)$ \\
H6 & 0.4147 & 0.5224 & 0.3865 & $0.091^{*}$ \\
H7 & 0.4880 & 0.6185 & 0.3024 & $0.091^{*}$ \\
H8 & 0.5755 & 0.5471 & 0.3943 & $0.091^{*}$ \\
C3 & $0.5148(3)$ & $0.1313(4)$ & $0.38747(18)$ & $0.0643(7)$ \\
H9 & 0.5211 & -0.0062 & 0.3597 & $0.097^{*}$ \\
H10 & 0.4328 & 0.1392 & 0.4203 & $0.097^{*}$ \\
H11 & 0.5940 & 0.1553 & 0.4309 & $0.097^{*}$ \\
C4 & $0.6306(3)$ & $0.2805(4)$ & $0.2525(2)$ & $0.0677(8)$ \\
H12 & 0.6329 & 0.1418 & 0.2254 & $0.102^{*}$ \\
H13 & 0.7143 & 0.3051 & 0.2916 & $0.102^{*}$ \\
H14 & 0.6217 & 0.3842 & 0.2032 & $0.102^{*}$ \\
& & & &
\end{tabular}

Atomic displacement parameters $\left(\AA^{2}\right)$

\begin{tabular}{lllllll}
\hline & $U^{11}$ & $U^{22}$ & $U^{33}$ & $U^{12}$ & $U^{13}$ & $U^{23}$ \\
\hline As1 & $0.03246(9)$ & $0.02705(9)$ & $0.02761(8)$ & $0.00106(11)$ & $0.00722(6)$ & $-0.00055(9)$ \\
O1 & $0.0324(6)$ & $0.0318(7)$ & $0.0448(7)$ & $0.0000(7)$ & $0.0090(5)$ & $0.0018(7)$ \\
O2 & $0.0539(10)$ & $0.0385(9)$ & $0.0368(8)$ & $0.0031(7)$ & $0.0162(7)$ & $0.0104(6)$ \\
O3 & $0.0507(9)$ & $0.0294(7)$ & $0.0433(8)$ & $0.0085(7)$ & $0.0196(6)$ & $0.0027(6)$ \\
O4 & $0.0452(9)$ & $0.0500(10)$ & $0.0474(9)$ & $0.0071(8)$ & $-0.0072(7)$ & $-0.0177(7)$ \\
N1 & $0.0389(8)$ & $0.0304(7)$ & $0.0312(7)$ & $0.0000(9)$ & $0.0053(6)$ & $0.0001(8)$ \\
C1 & $0.0392(11)$ & $0.0367(12)$ & $0.0371(10)$ & $-0.0018(8)$ & $-0.0017(8)$ & $0.0006(7)$ \\
C2 & $0.0685(18)$ & $0.0454(14)$ & $0.0640(16)$ & $-0.0033(13)$ & $-0.0198(13)$ & $-0.0125(12)$ \\
C3 & $0.0716(19)$ & $0.0592(17)$ & $0.0592(16)$ & $-0.0032(15)$ & $-0.0123(13)$ & $0.0228(14)$ \\
C4 & $0.0391(12)$ & $0.099(2)$ & $0.0653(16)$ & $-0.0058(14)$ & $0.0064(11)$ & $-0.0033(15)$ \\
\hline
\end{tabular}


Geometric parameters $(\AA, \stackrel{\circ}{)})$

\begin{tabular}{|c|c|c|c|}
\hline As1-O2 & $1.6412(13)$ & $\mathrm{C} 1-\mathrm{C} 4$ & $1.511(3)$ \\
\hline As $1-\mathrm{O} 1$ & $1.6687(13)$ & $\mathrm{C} 1-\mathrm{C} 2$ & $1.515(3)$ \\
\hline $\mathrm{As} 1-\mathrm{O} 3$ & $1.7061(13)$ & $\mathrm{C} 2-\mathrm{H} 6$ & 0.9600 \\
\hline As $1-\mathrm{O} 4$ & $1.7101(14)$ & $\mathrm{C} 2-\mathrm{H} 7$ & 0.9600 \\
\hline $\mathrm{O} 3-\mathrm{H} 1$ & 0.9710 & $\mathrm{C} 2-\mathrm{H} 8$ & 0.9600 \\
\hline $\mathrm{O} 4-\mathrm{H} 2$ & 0.9415 & $\mathrm{C} 3-\mathrm{H} 9$ & 0.9600 \\
\hline $\mathrm{N} 1-\mathrm{C} 1$ & $1.516(3)$ & $\mathrm{C} 3-\mathrm{H} 10$ & 0.9600 \\
\hline $\mathrm{N} 1-\mathrm{H} 3$ & 0.8900 & $\mathrm{C} 3-\mathrm{H} 11$ & 0.9600 \\
\hline $\mathrm{N} 1-\mathrm{H} 4$ & 0.8900 & $\mathrm{C} 4-\mathrm{H} 12$ & 0.9600 \\
\hline $\mathrm{N} 1-\mathrm{H} 5$ & 0.8900 & $\mathrm{C} 4-\mathrm{H} 13$ & 0.9600 \\
\hline $\mathrm{C} 1-\mathrm{C} 3$ & $1.506(3)$ & $\mathrm{C} 4-\mathrm{H} 14$ & 0.9600 \\
\hline $\mathrm{O} 2-\mathrm{As} 1-\mathrm{O} 1$ & $115.01(7)$ & $\mathrm{C} 2-\mathrm{C} 1-\mathrm{N} 1$ & $107.28(18)$ \\
\hline $\mathrm{O} 2-\mathrm{As} 1-\mathrm{O} 3$ & $106.49(7)$ & $\mathrm{C} 1-\mathrm{C} 2-\mathrm{H} 6$ & 109.5 \\
\hline $\mathrm{O} 1-\mathrm{As} 1-\mathrm{O} 3$ & $110.46(7)$ & $\mathrm{C} 1-\mathrm{C} 2-\mathrm{H} 7$ & 109.5 \\
\hline $\mathrm{O} 2-\mathrm{As} 1-\mathrm{O} 4$ & $111.36(8)$ & $\mathrm{H} 6-\mathrm{C} 2-\mathrm{H} 7$ & 109.5 \\
\hline $\mathrm{O} 1-\mathrm{As} 1-\mathrm{O} 4$ & $109.03(7)$ & $\mathrm{C} 1-\mathrm{C} 2-\mathrm{H} 8$ & 109.5 \\
\hline $\mathrm{O} 3-\mathrm{As} 1-\mathrm{O} 4$ & $103.90(7)$ & $\mathrm{H} 6-\mathrm{C} 2-\mathrm{H} 8$ & 109.5 \\
\hline $\mathrm{As} 1-\mathrm{O} 3-\mathrm{H} 1$ & 111.3 & $\mathrm{H} 7-\mathrm{C} 2-\mathrm{H} 8$ & 109.5 \\
\hline $\mathrm{As} 1-\mathrm{O} 4-\mathrm{H} 2$ & 113.1 & $\mathrm{C} 1-\mathrm{C} 3-\mathrm{H} 9$ & 109.5 \\
\hline $\mathrm{C} 1-\mathrm{N} 1-\mathrm{H} 3$ & 109.5 & $\mathrm{C} 1-\mathrm{C} 3-\mathrm{H} 10$ & 109.5 \\
\hline $\mathrm{C} 1-\mathrm{N} 1-\mathrm{H} 4$ & 109.5 & $\mathrm{H} 9-\mathrm{C} 3-\mathrm{H} 10$ & 109.5 \\
\hline $\mathrm{H} 3-\mathrm{N} 1-\mathrm{H} 4$ & 109.5 & $\mathrm{C} 1-\mathrm{C} 3-\mathrm{H} 11$ & 109.5 \\
\hline $\mathrm{C} 1-\mathrm{N} 1-\mathrm{H} 5$ & 109.5 & $\mathrm{H} 9-\mathrm{C} 3-\mathrm{H} 11$ & 109.5 \\
\hline $\mathrm{H} 3-\mathrm{N} 1-\mathrm{H} 5$ & 109.5 & $\mathrm{H} 10-\mathrm{C} 3-\mathrm{H} 11$ & 109.5 \\
\hline $\mathrm{H} 4-\mathrm{N} 1-\mathrm{H} 5$ & 109.5 & $\mathrm{C} 1-\mathrm{C} 4-\mathrm{H} 12$ & 109.5 \\
\hline $\mathrm{C} 3-\mathrm{C} 1-\mathrm{C} 4$ & $111.7(2)$ & $\mathrm{C} 1-\mathrm{C} 4-\mathrm{H} 13$ & 109.5 \\
\hline $\mathrm{C} 3-\mathrm{C} 1-\mathrm{C} 2$ & $111.0(2)$ & $\mathrm{H} 12-\mathrm{C} 4-\mathrm{H} 13$ & 109.5 \\
\hline $\mathrm{C} 4-\mathrm{C} 1-\mathrm{C} 2$ & $112.1(2)$ & $\mathrm{C} 1-\mathrm{C} 4-\mathrm{H} 14$ & 109.5 \\
\hline $\mathrm{C} 3-\mathrm{C} 1-\mathrm{N} 1$ & $107.39(18)$ & $\mathrm{H} 12-\mathrm{C} 4-\mathrm{H} 14$ & 109.5 \\
\hline $\mathrm{C} 4-\mathrm{C} 1-\mathrm{N} 1$ & $107.17(18)$ & $\mathrm{H} 13-\mathrm{C} 4-\mathrm{H} 14$ & 109.5 \\
\hline
\end{tabular}

Hydrogen-bond geometry $\left(\AA,{ }^{\circ}\right)$

\begin{tabular}{lllll}
\hline$D-\mathrm{H} \cdots A$ & $D-\mathrm{H}$ & $\mathrm{H} \cdots A$ & $D \cdots A$ & $D-\mathrm{H} \cdots A$ \\
\hline $\mathrm{O} 3-\mathrm{H} 1 \cdots \mathrm{O} 1^{\mathrm{i}}$ & 0.97 & 1.62 & $2.5752(19)$ & 166 \\
$\mathrm{O} 4-\mathrm{H} 2 \cdots \mathrm{O} 1^{\mathrm{ii}}$ & 0.94 & 1.74 & $2.6763(19)$ & 172 \\
$\mathrm{~N} 1-\mathrm{H} 3 \cdots \mathrm{O} 2$ & 0.89 & 1.92 & $2.801(2)$ & 168 \\
$\mathrm{~N} 1-\mathrm{H} 4 \cdots \mathrm{O} 2^{\mathrm{iii}}$ & 0.89 & 1.88 & $2.765(2)$ & 173 \\
$\mathrm{~N} 1-\mathrm{H} 5 \cdots 3^{\mathrm{iv}}$ & 0.89 & 2.07 & $2.944(2)$ & 166 \\
\hline
\end{tabular}

Symmetry codes: (i) $-x,-y,-z+1$; (ii) $-x,-y+1,-z+1$; (iii) $-x+1 / 2, y-1 / 2,-z+1 / 2$; (iv) $-x+1 / 2, y+1 / 2,-z+1 / 2$. 